tivity and a reduction in the growth rate; it does not, however, induce concomitant morphological differentiation.

Waymire et al., who were, of course, out to establish that cAMP, not butyrate, has a role in the regulation of the activity of tyrosine hydroxylase activity in these cells, must have been abashed by this surprising observation. They seem, however, to have convinced themselves that the level of tyrosine hydroxylase activity rises in parallel with increasing intracellular concentrations of cAMP by showing that other substituted cAMPs as well as papaverine induce significant increases in the activity of this enzyme and in some cases concomitant morphological differentiation. They also obtained data which suggest that the increased activity of this enzyme results from synthesis of new enzyme molecules. But the awkward fact that sodium butyrate induces an increase in the activity of tyrosine hydroxylase remains. Not everyone will be satisfied with the suggestion that sodium butyrate may be mimicking the action of neurobiologically active molecules such as $\gamma$ amino and $\gamma$ hydroxy butyric acids but no doubt cells in more than one laboratory are about to taste sodium butyrate.

Prasad and Sheppard (Exp. Cell Res., 73, 437; 1972) have also recently reported that papaverine and two other inhibitors of phosphodiesterases induce the formation of axon-like processes by neuroblastoma cells. This response to these inhibitors, which for the first $24 \mathrm{~h}$ is reversible but thereafter is irreversible, apparently precedes the maximal inhibition of cell division. Prasad and Sheppard conclude, therefore, that the morphological differentiation is probably a direct response to the inhibitors of phosphodiesterases rather than a secondary response to the inhibition of cell division. But they were obviously in a justifiably cautious mood when they wrote this report, for after drawing attention to the fact that these inhibitors might be expected to cause an increase in the intracellular concentration of cAMP and having noted that $\mathrm{Bu}_{2}$-cAMP and prostaglandins also induce the formation of axonal processes, they caution that "these data, as yet, are inadequate to suggest any direct role of cyclic AMP in the differentiation of neuroblastoma cells".

Willingham, Johnson and Pastan (Biochem. Biophys. Res. Commun., 48, $743 ; 1972$ ), by contrast, have not demurred at offering a hostage to fortune. They have been investigating the effects of adding $\mathrm{Bu}_{2}$-cAMP to synchronized cultures of Swiss 3T3 and $\mathrm{BALB} / \mathrm{C}$ 3T3 mouse fibroblasts. $\mathrm{Bu}_{2}$-cAMP blocked mitosis when added after the cells had entered $S$ phase even though the round of DNA replication was completed; it also blocked entry into $S$ phase when fed to the cells continuously after replating. In short, $\mathrm{Bu}_{2}$-cAMP can block cells in the $\mathrm{G} 1$ or $\mathrm{G} 2$ phases. No one will quarrel with those conclusions, but Willingham et al. further claim that late in $\mathbf{G 1}$ phase high concentrations of cyclic AMP hasten the onset of DNA synthesis. Their data, however, show that while Swiss 3T3 cells exhibit this response BALB/C 3T3 cells do not, which means, to say the least of it, that this putative positive regulatory function of cAMP is not universal even to cultivated mouse fibroblasts.

\section{SOLID STATE \\ Shocked Ceramics}

AN interesting correlation between radiation damage and the effects of explosive shock waves is drawn by R. L. Nelson, of the United Kingdom Atomic Energy Authority, Harwell, and D. B. Hartley, of the Explosives Research and Development Establishment, Waltham Abbey, in the latest issue of the Journal of the Chemical Society, Dalton Transactions (1596; 1972). Nelson and Hartley compared the reactions of several crystalline ceramics to explosive shocking and high energy radiation by means of electron spin resonance and diffuse reflectance measurements, and they also considered surface area changes and such physical properties as colour changes.

The importance of a completely ordered structure in crystalline materials has been appreciated for many years, and the properties of metals, for example, are known to be correlated with the density of atomic defects or dislocations. Recently, however, the creation of small scale defects and much larger disturbances of the crystal lattice have assumed some practical importance, in particular in studies of the properties of reactor cores.

Of the three ceramics studied by Nelson and Hartley-magnesia, zinc oxide and silica-only the magnesium oxide showed similar behaviour when it was irradiated with high energy elec. trons or neutrons and when it was subjected to a shock wave. The silica displayed no correlation between the two processes, whereas only moderate similarities in behaviour emerged from a study of the zinc oxide.

It is significant that in this series of experiments the material with the lowest melting point showed least similarity between damage induced by shock waves and by radiation. It has been suggested by Symons (J. Chem. Soc., $A, 1648 ; 1971)$ that no correlations exist between defects formed in solid materials by explosive shock waves and those formed by radiation, but the

\title{
A Precursor of Immunoglobulin Light Chain
}

Although it is becoming apparent that many, if not all, mammalian proteins are initiated with methionine, studies of amino-acid sequence on the $\mathrm{N}$-terminal ends of immunoglobulins have failed to detect this initial methionine residue. With the report of Milstein, Brownlee, Harrison and Mathews in Nature New Biology next Wednesday (September 27) much of the speculation concerning the possible post-synthetic cleavage of the individual heavy and light chains of immunoglobulins will be ended.

By using purified mRNA, polyribosomes or microsomes from myeloma MOPC 21 as the source of mRNA, they have shown that in a reticulocyte cellfree system, the light chain product obtained from mRNA or polyribosomes has a molecular weight about 1,500 daltons greater than the light chain obtained from a microsomal-primed preparation, or from the in vivo light chain. Although tryptic digests of the larger molecule (designated $P$ ) have established that it contains some peptides identical to light chain synthesized in vivo, differences in the $\mathrm{N}$-terminal peptide are seen, establishing the location of at least part of the excess material, presumably amino-acids, at the N-terminal end. Sequence studies of this new $\mathrm{N}$-terminal sequence will be anxiously awaited.

One of the more striking features of the system is the requirement that membrane should be present at the time of synthesis of $P$ in order to effect the ultimate cleavage to light chain. It should be noted that the specificity of cleavage appears not to be confined to membranes from myeloma cells as membraneous material from Krebs ascites cells seems to work equally well.

Although the function of the extra amino-acids is not clearly understood, the hypothesis most attractive to Milstein and his colleagues is that, following initiation of protein synthesis on free ribosomes, the peptide is used to determine which ribosomes become membrane-bound. Alternatively the modified $\mathrm{N}$-terminus may function to initiate an exit through the membrane for the protein being synthesized.

Whether or not the N-terminal sequence is peculiar to immunoglobulin light chains or whether it indeed functions in a more general manner for all secreted proteins will have to await the demonstration and characterization of similar peptides from both additional immunoglobulin chains as well as other proteins. 